\title{
Natural dipeptidyl peptidase-4 inhibitor Terminalia arjuna mitigates myocardial infarction co-existing with diabetes in experimental rats
}

\begin{abstract}
Background: Large pool of diabetic patients have co-existing cardiovascular diseases, DPP-4 inhibitors may represent novel and promising antidiabetic agents with potential cardiovascular benefits. However they are expensive drugs and recently have been associated with a number of unacceptable adverse effects. Terminalia arjuna have been reported to possess antidiabetic activity, its cardioprotective effects in the setting of diabetes has not been studied so far. DPP-4 based therapeutics may represent novel antidiabetic drug, the cardioprotective actions of which may translate into demonstrable therapeutic benefits in diabetes with co-existing cardiovascular diseases. With this point of view the present study was designed.
\end{abstract}

Material and methods: The study is experimental study conducted in department of pharmacology, MGM Medical College, Navi Mumbai. The rats were randomly allocated in various group for 5 week. STZ $45 \mathrm{mg} / \mathrm{kg}$ administered on $\mathrm{I}^{\text {st }}$ week to induce the diabetes and Isoproterenol $85 \mathrm{mg} / \mathrm{kg}$ administered on 2 subsequent days before scarification of rats to induce myocardial infarction. Blood glucose $>200 \mathrm{mg} / \mathrm{dl}$ on end of the $\mathrm{I}^{\text {st }}$ week confirm the diabetes. Hydoalcoholic extract of Terminalia arjuna was fed orally from $2^{\text {nd }}$ week to $5^{\text {th }}$ week and rats were scarified for biochemical and histopathological parameter.

Results: Efficacy of Terminalia arjuna (TA) was observed on various parameter of diabetes with cardiovascular complication. TA treatment showed restoration of body weight as compare with control group. The treatment with TA reduced hyperglycemia as compare with (Diabetic ISP control group) D-ISP group.Vildagliptin (VIL) treatment showed superior effect in controlling hyperglycemia as compare with TA. The VIL \& TA treatment group rats showed significantly reduced heart to body weight ratio as compared to D-ISP rats. VIL \& TA treated group significantly reversed the STZ/ISP induced increase in CPK-MB, hsCRP levels and marked protection against cardiac damage was observed as indicated by decrease in serum CPK-MB isoenzyme, hs-CRP in treated rats as compared to D-ISP group rats.VIL \& TA treated rats showed significant reduction in serum DPP-4 levels as compared to D-ISP rats. Significant cardioprotection as indicated by positive correlation between cardiac marker CPK-MB and serum DPP-4 in VIL $(r=0.899 ; p<0.01)$, TA $(r=0.848 ; p<$ $0.05)$ groups was also confirmed by histopathological assessment. As per histopathological and biochemical report, TA treatment does not show delineated effect in pancreas, liver and kidney.

Conclusion: Terminalia arjuna demonstrated beneficial effects in experimental model of myocardial infarction co-existing with diabetes.
Volume 5 Issue 2 - 2018

\author{
Manjusha K Borde,' Ipseeta Ray Mohanty, \\ Ujwala Maheshwari, ${ }^{2}$ Rajesh Kumar Suman, ${ }^{3}$ \\ YA Deshmukh² \\ 'Department of Pharmacology, Sinhgad Dental College \& \\ Hospital, India \\ ${ }^{2}$ Department of Pharmacology, Pathology, MGM Medical \\ College, India \\ ${ }^{3}$ Department of Pharmacology, Hind Institute of Medical \\ Sciences, India
}

Correspondence: Rajesh Kumar Suman, Assistant professor Department of pharmacology, Hind Institute of medical sciences, Mau, Ataria, Sitapur, Lucknow, India,

Email rajesh_suman1986@hotmail.com, rajeshsuman2043@gmail.com

Received: January 21, 2018 | Published: March 28, 2018

\section{Introduction}

Diabetes mellitus (DM) and ensuing cardiovascular (CV) complications have arisen as the epidemic of the early $21^{\text {st }}$ century. Diabetes mellitus remains a profound risk factor for cardiovascular disease. Excess mortality in type $2 \mathrm{DM}$ is largely related to an increased incidence of CV disease with approximately $75 \%$ of deaths in people with diabetes attributable to stroke, myocardial infarction (MI) and peripheral arterial disease. ${ }^{1,2}$ Management of cardiovascular risk is an essential aspect of diabetes care and acceptable CV risk is a requirement for Antidiabetes medications. DPP-4 inhibitors are a novel class of oral hypoglycemic agents, widely used for the treatment of type 2 diabetes mellitus (T2DM). Besides established antidiabetic effects, several studies have reported the cardioprotective benefits of DPP-4 inhibitors via GLP-1 dependent and independent pathways. Studies documented that, DPP-4 inhibitors improve several cardiovascular risk factors: they improve glucose control (by reducing the risk of post prandial and fasting hyperglycemia), weight neutral, lower blood pressure, improve dyslipemia, reduce inflammatory markers, diminish oxidative stress, improve endothelial functions and reduce platelet aggregation in patients with T2DM., ${ }^{3,4}$ In Zucker diabetic fatty rats, a genetic rodent model for type 2 diabetes, the inhibition of DPP-4 corrected glycemic dysmetabolism, hypertriglyceridemia, inflammation and hypertension. ${ }^{5}$ Since a large pool of diabetic patients have co-existing cardiovascular diseases, DPP-4 inhibitors may represent novel and promising ant diabetic agents with potential cardiovascular benefits. Thus, DPP-4 inhibitors are a promising new therapeutic approach for the management of type 2 diabetes. However they are expensive drugs and recently have been associated with a number of unacceptable adverse effects. ${ }^{6-9}$ 
In this scenario, if novel DPP-4 inhibitors from alternative sources are developed, that share the advantages of DPP-4 inhibition but at the same time overcome the limitations of the currently available synthetic DPP-4 inhibitors, it would be very beneficial. Terminalia arjuna (Combretaceae), commonly known as Arjuna, has been used in Indian system of medicine for over three centuries. The thick, white to pinkish gray bark has a long ethnomedicinal history including cancer treatment; cardioprotective, hypotensive, hypolipidemic and wound healing activity. ${ }^{10}$ Medicinal plants have been observed to possess numerous activities with regard to hypoglycemic and cardiovascular disorder. Despite the wide usage of medicinal herbs for several pathological conditions in Ayurveda, few systemically designed studies are available to validate their therapeutic effects. Although Terminalia arjuna have been reported to possess antidiabetic activity, ${ }^{11-13}$ its cardioprotective effects in the setting of diabetes has not been studied so far. DPP-4 based therapeutics may represent novel anti-diabetic drug, the cardioprotective actions of which may translate into demonstrable therapeutic benefits in diabetes with co-existing cardiovascular diseases. With this point of view the present study was designed. The results of present study will provide preliminary experimental evidence on the potential therapeutic benefits of using natural DPP-4 inhibitors in the setting of diabetes co-existing with cardiovascular diseases. Various parameters like anti-diabetic, cardioprotective, anti-inflammatory, antioxidant, DPP-4 pathway, safety \{pancreas, liver and kidney function\} and histopathological indices of injury were evaluated in experimental groups.

\section{Materials and methods}

\section{Chemicals and test drugs}

Streptozotocin (STZ) and Isoproterenol (ISP) were procured from Sigma Chemicals St Louis, USA. The test drugs Vildagliptin was obtained as gift sample. The hydro-alcoholic dried standardized extract of Terminalia arjuna were procured from Sanat Pharmaceutical, New Delhi. All other chemicals and reagents used were of analytical grade.

\section{Experimental animals}

The study has been conducted at MGM medical college and hospital Navi Mumbai. Wistar rats were procured from Bombay Veterinary College, BVC Campus Road, Parel, Mumbai. Adult male Wistar rats, 10 to 12 weeks old, weighing 150 to 200 gm were used in the study. Rats were housed in the Animal Facility of Mahatma Gandhi Mission Medical College, Navi Mumbai, Indiain polyacrylic cages $(38 \times 23 \times 10 \mathrm{~cm})$ under standard laboratory conditions. The animals were allowed free excess to standard diet, tap water adlibitum and allowed to acclimatize for one week before the experiments. The study protocol was approved by the Institutional Animal Ethics Committee and conforms to the Committee for the Purpose of Control and Supervision of Experiments on Animals and Indian National Science Academy and Guidelines for the Use and Care of Experimental Animals in Research.

\section{Experimental model of myocardial infarction in the setting of diabetes mellitus}

Male wistar rats weighing 150- 200 gm was used for the study. Type II Diabetes was induced in rats by a single Streptozotocin injection $(45 \mathrm{mg} / \mathrm{kg}$ body wt, intraperitoneal \{i.p.\} dissolved in 0.01 $\mathrm{M}$ cold citrate buffer, $\mathrm{pH} 4.5$ ) in overnight fasted rats. Serum glucose estimations (blood sugar $>200 \mathrm{mg} / \mathrm{dL}$ ) were undertaken periodically (day 0,3 and 7) from the tail vein to confirm the production of diabetes mellitus. Animals showing fasting blood glucose higher than $200 \mathrm{mg} / \mathrm{dL}$ were considered as diabetic. Myocardial infarction (MI) was produced by Isoproterenol injection $(85 \mathrm{mg} / \mathrm{kg}$ body weight, subcutaneous $\{$ s.c. $\}$ injection dissolved in normal saline) 24 and 48 $\mathrm{h}$ prior to scarification ( $5^{\text {th }}$ week). At the end of experimental period, rats were sacrificed for further biochemical investigations as well as histopathological evaluation.

\section{Experimental groups and treatment protocol}

Group 1: Normal control (NC): In Normal Control group, rats were administered distilled water per orally using a feeding cannula for study period of 5 weeks.

Group 2: Diabetic ISP control(D-ISP): The rats were administered distilled water per orally using a feeding cannula for study period of 5 weeks. Streptozotocin $(45 \mathrm{mg} / \mathrm{kg}$ body weight, i.p.) was injected to induce diabetes at 0 week and challenged with Isoproterenol $(85 \mathrm{mg} /$ $\mathrm{kg}$ body weight sc.) 24 and $48 \mathrm{~h}$ prior to scarification.

Group 3: Vildagliptin (VIL): Vildagliptin $(10 \mathrm{mg} / \mathrm{kg})$ was administered orally using a feeding cannula from $1^{\text {st }}$ to $5^{\text {th }}$ week (4 weeks). The Streptozotocin ( $45 \mathrm{mg} / \mathrm{kg}$ body weight, i.p.) was injected to induce diabetes at 0 week. Subsequently the rats were challenged with Isoproterenol $(85 \mathrm{mg} / \mathrm{kg}$ body weight sc) 24 and $48 \mathrm{~h}$ prior to sacrification.

Group 4: Terminalia arjuna (TA): Terminalia arjuna $(500 \mathrm{mg} /$ $\mathrm{kg}$ ) was administered orally using a feeding cannula from $1^{\text {st }}$ to $5^{\text {th }}$ week (4 weeks). The Streptozotocin $(45 \mathrm{mg} / \mathrm{kg}$ body weight, i.p.) was injected to induce diabetes at 0 week. Subsequently the rats were challenged with Isoproterenol ( $85 \mathrm{mg} / \mathrm{kg}$ body weight sc) 24 and $48 \mathrm{~h}$ prior to scarification.

\section{Evaluation parameters}

\section{Assessment of body weight changes}

Each rat was weighed individually twice, first at the beginning of the experiment (initial weight) and second, $24 \mathrm{~h}$ after the administration of the last dose of either drug (final weight). The difference in body weight of each rat was calculated and expressed as percentage change according to the following:

$\%$ change in body weight $=$ Final weight - Initial weight $\mathrm{X}$ $100 /$ Initial weight

\section{Biochemical Parameters}

Biochemical parameters were estimated using AU 480 autoanalyzer Backman coulter, Germany. The rat blood samples of all experimental groups were collected from the retro-orbital plexus under light ketamine anesthesia $\left(40 \mathrm{mg} / \mathrm{kg}\right.$ i.p.) at $0,1^{\text {st }}$ and $3^{\text {rd }}$ week for estimation of blood glucose and creatinine phosphokinase (CPK$\mathrm{MB})$. In addition, after the completion of the experimental duration ( $5^{\text {th }}$ weeks), Serum was used for the determination of the biochemical parameters blood glucose ${ }^{14} \mathrm{HbA} 1 \mathrm{c},{ }^{15}$ creatinine phosphokinase (CPKMB),${ }^{16}$ serum DPP-4, ${ }^{17}$ high sensitive C-reactive protein (hs-CRP),${ }^{18}$ pancreatic lipase, ${ }^{19}$ serum glutamate pyruvate transaminase (SGPT), ${ }^{20}$ creatinine ${ }^{21}$ by AU480 auto analyzer Backman coulter, Germany or ELISA kits in the Pathology (NABL accredited) and Pharmacology laboratory. 


\section{Histopathological studies}

At the end of the experiment ( 5 weeks), the animals were sacrificed. The heart, pancreas, liver and kidney were immediately fixed in $10 \%$ buffered neutral formalin solution. The tissues were carefully embedded in molten paraffin with the help of metallic blocks, covered with flexible plastic moulds and kept under freezing plates to allow the paraffin to solidify. Cross sections ( $5 \mu \mathrm{m}$ thick) of the fixed tissues were cut. These sections were stained with hematoxyline and eosin and visualized under light microscope to study the microscopic architecture of the tissues. The investigator performing the histological evaluation was blind to biochemical results and to treatment allocation. (H\&E 40×)

\section{Statistical analysis}

All numerical data in text, figures and tables were expressed as the mean $+\mathrm{SD}$. Statistical analysis was performed by student $t$ test and One-way analysis of variance (ANOVA). Spearman correlation coefficient was used to determine the relationship between different variables Differences were considered statistically significant at $\mathrm{p}<0.05$.

\section{Results}

\section{General observations and assessment of body weight changes}

Body weight of rats in all the groups was recorded every week and the percentage change in body weight was calculated after $5^{\text {th }}$ week The percentage change in body weight in NC group rats at the end of $5^{\text {th }}$ week was found to be 14.45 , D-ISP;-10.03, VIL;-1.78, TA; 4.97. The D-ISP group rats showed significant decrease in body weight (\%) as compared with $\mathrm{NC}$, VIL, TA treated groups. TA treatment showed significant $(\mathrm{p}<0.01)$ restoration in body weight as compared with VIL group (Figure 1).

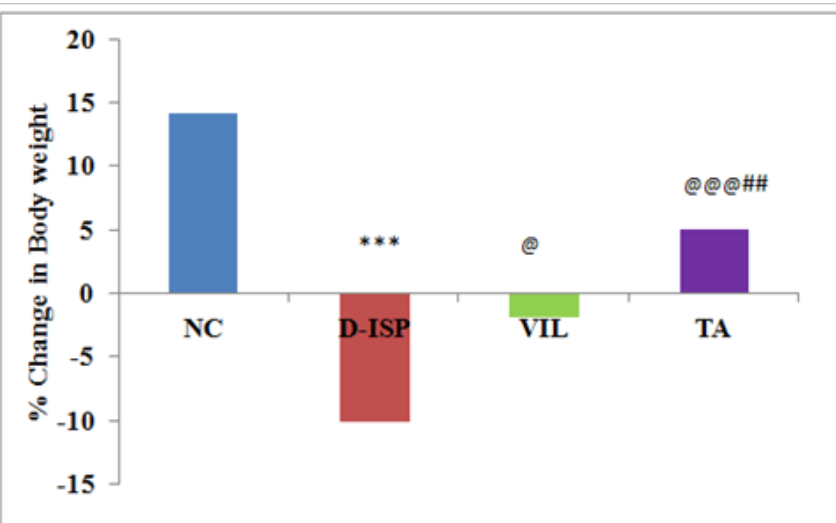

Figure 1 Body weight changes among various experimental groups.

Values are expressed as mean $\pm \mathrm{S} \mathrm{D}$.

***p $<0.001$ NC VS D-ISP;@@@ @ $<0.001$ @ p $<0.05$ D-ISP VS VIL, TA; $\# \# \mathrm{p}<0.01$ TA VS VIL

NC, Normal control ( $\mathrm{n}=8)$; D-ISP, Diabetic-isoproterenol control ( $\mathrm{n}=9)$; VIL, Vildagliptin $(\mathrm{n}=8)$; TA, Terminalia arjuna $(\mathrm{n}=7)$

\section{Comparative cardioprotective efficacy of natural and synthetic DPP-4 inhibitors}

Diabetic parameter: Hyperglycemia induced by Streptozotocin was maintained throughout the study period as evidenced by persistent hyperglycemia throughout the study duration. There was a significant $(p<0.001)$ increase in blood glucose and glycosylated hemoglobin levels in D-ISP group rats as compared to NC group. Oral feeding of TA $(500 \mathrm{mg} / \mathrm{kg})$ significantly restored $(\mathrm{p}<0.001)$ the elevated blood glucose levels as compared to D-ISP group rats. Similarly glycosylated hemoglobin was also reduced in TA $(500 \mathrm{mg} /$ $\mathrm{kg}$ ) treatment group as compared to D-ISP group rats. As shown in figure, VIL $(10 \mathrm{mg} / \mathrm{kg})$ as compared to D-ISP group was significantly decreased the high glucose and glycosylated hemoglobin followed by TA $(500 \mathrm{mg} / \mathrm{kg})$ treatment. The anti diabetic efficacy of VIL was found to be superior to TA therapy (Figure 2) (Figure 3).

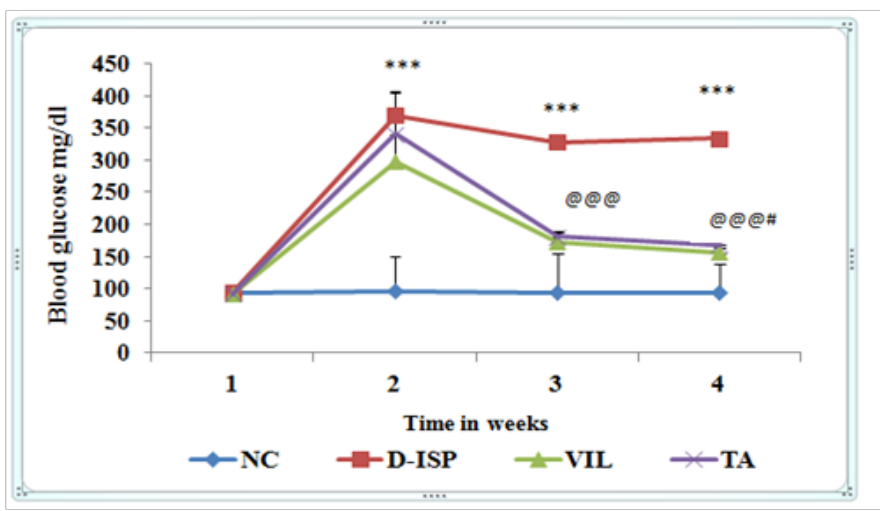

Figure 2 Time course changes in blood glucose levels among various experimental groups.

Values are expressed as mean $\pm \mathrm{SD}$

*** P<0.00I NCVS D-ISP; @@@P<0.00I D-ISPVSVIL,TA, \#p<0.05 TA VSVIL.

NC, Normal control (n=8); D-ISP, Diabetic-isoproterenol control $(n=9)$; VIL, Vildagliptin $(n=8)$; TA, Terminalia arjuna $(n=7)$

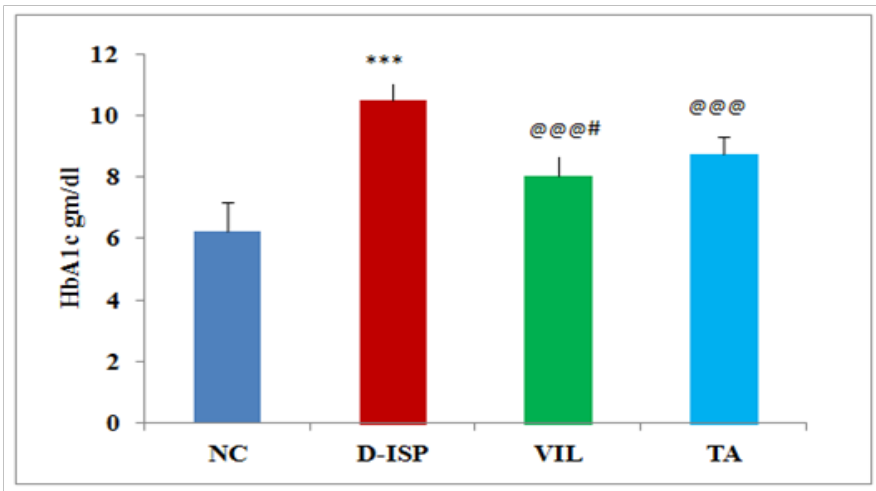

Figure 3 Glycosylated hemoglobin among various experimental groups. *** P<0.00I NCVS D-ISP; @@@p<0.00I D-ISPVSVIL,TA, \#p<0.05 TAVSVIL.

NC, Normal control $(n=8)$; D-ISP, Diabetic-isoproterenol control $(n=9)$; VIL, Vildagliptin $(n=8) ; T A$, Terminalia arjuna $(n=7)$

Cardiac parameters: The D-ISP control rats showed significantly increase in $(p<0.001)$ heart to body weight ratio as compared to NC rats. 
The VIL $(10 \mathrm{mg} / \mathrm{kg})(\mathrm{p}<0.01) \& \mathrm{TA}(500 \mathrm{mg} / \mathrm{kg})(\mathrm{p}<0.05)$ treatment group rats showed significantly reduced heart to body weight ratio as compared to D-ISP rats. VIL $(10 \mathrm{mg} / \mathrm{kg})$ treatment significantly reduced $(\mathrm{p}<0.05)$ heart to body weight ratio as compared to TA $(500$ $\mathrm{mg} / \mathrm{kg}$ ) (Figure 4). There was a significant increase in cardiac markers of injury CPK-MB $(\mathrm{p}<0.001)$, hs-CRP $(\mathrm{p}<0.01)$ level in D-ISP rats as compared to NC group at $5^{\text {th }}$ week of study after ISP challenge. VIL $(10 \mathrm{mg} / \mathrm{kg}) \& \mathrm{TA}(500 \mathrm{mg} / \mathrm{kg})$ treated group significantly reversed the STZ/ISP induced increase in CPK-MB $(\mathrm{p}<0.001)$, hs-CRP $(\mathrm{p}<0.05)$ levels at $5^{\text {th }}$ week. A marked protection against cardiac damage was observed as indicated by decrease in serum CPK-MB isoenzyme, hsCRP in treated rats as compared to D-ISP group rats. However, the cardioprotective efficacy of the marketed synthetic DPP-4 inhibitor: Vildagliptin was found to be superior to TA $(p<0.01)$ (Table 1). The serum DPP-4 levels $(\mathrm{p}<0.001)$ increased significantly in D-ISP group rats as compared to NC group rats. VIL $(10 \mathrm{mg} / \mathrm{kg}) \&$ TA $(500 \mathrm{mg} /$ $\mathrm{kg}$ ) treated rats showed significant reduction in serum DPP-4 levels as compared to D-ISP rats. The VIL $(10 \mathrm{mg} / \mathrm{kg})$ treated rats showed significant reduction $(\mathrm{p}<0.01)$ in serum DPP-4 levels as compared to $\mathrm{TA}(500 \mathrm{mg} / \mathrm{kg})$ treated rats. Significant cardioprotection as indicated by positive correlation between cardiac marker CPK-MB and serum DPP-4 in VIL $(\mathrm{r}=0.899 ; \mathrm{p}<0.01)$, TA $(\mathrm{r}=0.848 ; \mathrm{p}<0.05)$ groups was also confirmed by histopathological assessment (Table 1), (Figure 5) (Figure 6).

Table I Cardiac parameters and serum DPP-4 levels among various experimental groups

\begin{tabular}{lllllll}
\hline SN & Parameters & Variable & NC & D-ISP & VIL & TA \\
\hline I & $\begin{array}{l}\text { Cardiac } \\
\text { parameters }\end{array}$ & CPK-MB $(\mathrm{U} / \mathrm{L})$ & $1565.12 \pm 292.07$ & $5424.28 \pm 837.73 * * *$ & $2311.25 \pm 253.96 @ @ @ \#$ & $2865.71 \pm 119.7 @ @ @$ \\
2 & & hs-CRP $(\mathrm{mg} / \mathrm{dl})$ & $0.86 \pm 0.11$ & $1.9 \pm 0.5 * *$ & $0.91 \pm 0.1 @ @ \#$ & $1.2 \pm 0.22 @$ \\
3 & DPP-4 Pathway & $\begin{array}{l}\text { Serum DPP-4 } \\
\text { (microunit } / \mathrm{ml})\end{array}$ & $4.76 \pm 0.43$ & $38.25 \pm 4.25 * * *$ & $12.22 \pm 1.35 @ @ @ \# \#$ & $18.42 \pm 2.04 @ @ @$ \\
\hline
\end{tabular}

Values are expressed as mean $\pm \mathrm{SD}$.

*** $\mathrm{p}<0.001$ NCVS D-ISP;

@@@ $<<0.001$;

@@ $\mathrm{p}<0.01$,

@ $\mathrm{p}<0.05 \mathrm{D}-$ ISPVSVIL,TA;

$\# \mathrm{p}<0.01$, \#<0.05VILVSTA.

CPK-MB, Creatinine phosphokinase-MB; hs-CRP, High sensitive reactive protein; DPP-4, Dipeptidyl peptidase-4; NC, Normal control ( $n=8$ ); D-ISP, DiabeticIsoproterenol control ( $n=9)$;VIL,Vildagliptin $(n=8) ; T A$, Terminalia arjuna $(n=7)$

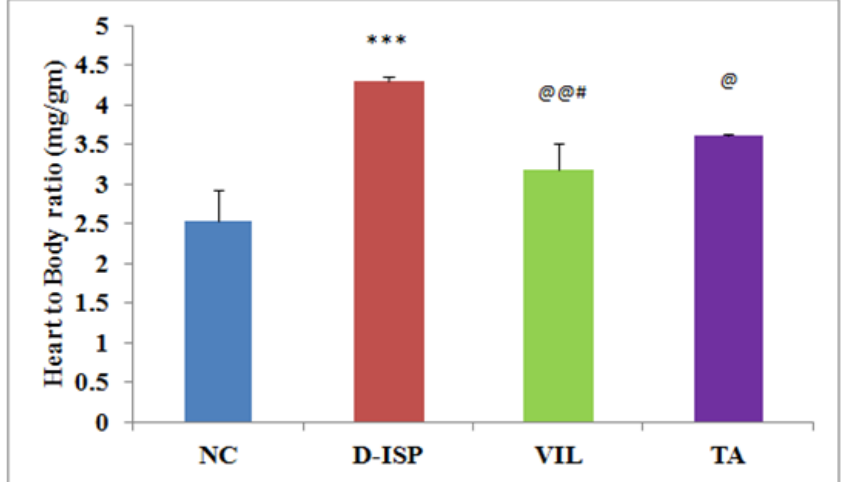

Figure 4 Heart to body weight ratio among various experimental groups.

Values are expressed as mean $\pm \mathrm{SD}$

*** P<0.00I NCVS D-ISP; @@@p<0.00I D-ISPVSVIL,TA,\#p<0.05 TAVSVIL.

NC, Normal control ( $n=8)$; D-ISP, Diabetic-isoproterenol control $(n=9)$; VIL, Vildagliptin $(n=8) ; T A$, Terminalia arjuna $(n=7)$

\section{Histopathological assessment}

\section{Histopathological section of myocardium}

Photomicrograph of NC group rat heart revealed the non-infracted architecture of the myocardium (Plate A). In contrast, D-ISP group rat heart showed fatty infiltration in myocardial cells, hemorrhage, and marked edema, separation of myofibers, congested blood vessels and inflammation as compared to the $\mathrm{NC}$ group (Plate B). In the VIL treatment group rats, occasional focal myofibers loss, less inflammation, necrosis and edema was observed (Plate C). In the TA treatment group rats, occasional focal myofibers loss, inflammation, necrosis and edema was observed (Plate D) (Figure 7).

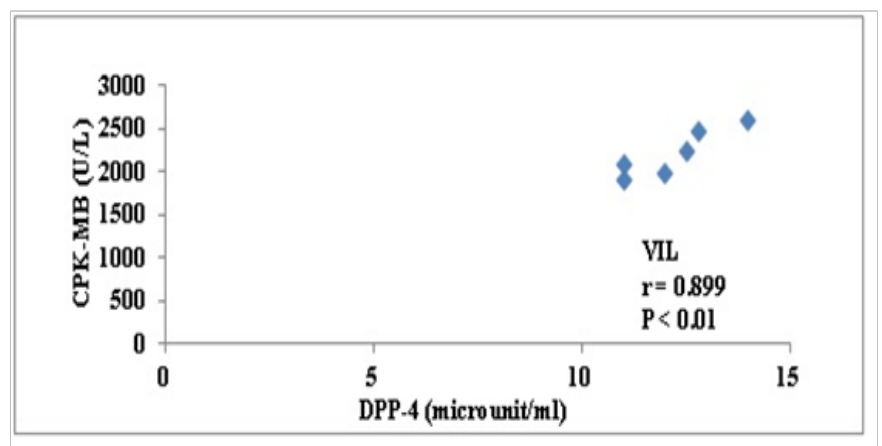

Figure 5 Correlation between CPK-MB and serum DPP-4 of Vildagliptin treated group.

CPK-MB, Creatinine phosphokinase-MB; DPP-4, Dipeptidyl peptidase-4.

Positive correlation between CPK-MB and serum DPP-4; $r=0.899, P<0.0$ I

\section{Histopathological section of pancreas}

Photomicrograph of pancreatic sections of $\mathrm{NC}$ rats showed an 
organized pattern and normal architecture of islets of langerhans and the beta cells (Plate A). In contrast, the pancreas of D-ISP group rat showed severe degenerative changes in the pancreatic islets, damaged islets of langerhans, reduced beta cell mass and the atrophy of beta cells with the loss of few nucleus and cytoplasm and inflammatory infiltration (Plate B). Treatment group rats pancreas showed improved beta cell mass, less fibrosis, less inflammatory infiltration and hemorrhage as compared to D-ISP group (Plate C,D) (Figure 8), (Table 2).

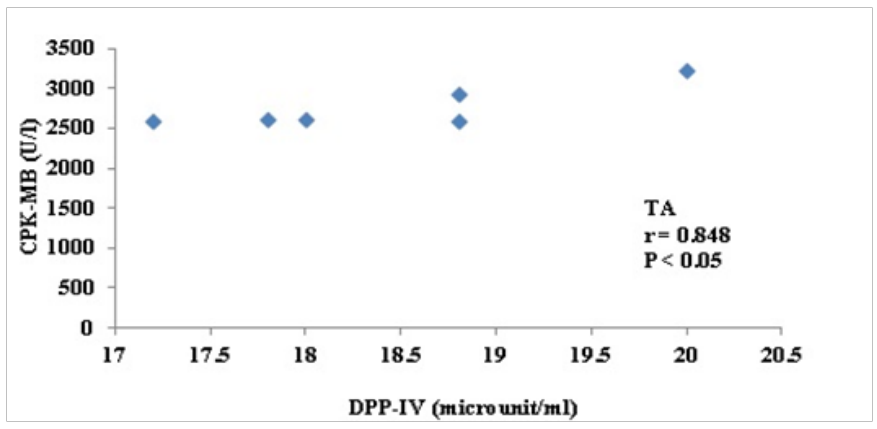

Figure 6 Correlation between CPK-MB and serum DPP-4 of Terminalia arjuna treated group.

CPK-MB, Creatinine phosphokinase-MB; DPP-4, Dipeptidyl peptidase-4.

Positive correlation between CPK-MB and serum DPP-4; $r=0.899, \mathrm{P}<0.0 \mathrm{I}$

\section{Safety of natural DPP-4 inhibitors: terminalia arjuna therapy}

As seen from the table 2, it was found that the in D-ISP group a significant elevation in the levels of pancreatic lipase (U/L) $(p<0.001)$, SGPT $(\mathrm{U} / \mathrm{L}) \quad(\mathrm{p}<0.001)$ and Creatinine $(\mathrm{mg} / \mathrm{dl}) \quad(\mathrm{p}<0.001)$ was observed at $5^{\text {th }}$ week compared to $\mathrm{NC}$ group. The treatment groups did not adversely affect the pancreatic, liver and kidney function in myocardial infarction co-existing with diabetes rats, as evidenced by pancreatic, hepatic and renal biochemical markers of injury as well as histopathological studies.

\section{Safety of natural DPP-4 inhibitors: terminalia arjuna therapy}

As seen from the table 2, it was found that the in D-ISP group a significant elevation in the levels of pancreatic lipase $(\mathrm{U} / \mathrm{L})(\mathrm{p}<0.001)$, SGPT $(\mathrm{U} / \mathrm{L}) \quad(\mathrm{p}<0.001)$ and Creatinine $(\mathrm{mg} / \mathrm{dl}) \quad(\mathrm{p}<0.001)$ was observed at $5^{\text {th }}$ week compared to $\mathrm{NC}$ group. The treatment groups did not adversely affect the pancreatic, liver and kidney function in myocardial infarction co-existing with diabetes rats, as evidenced by pancreatic, hepatic and renal biochemical markers of injury as well as histopathological studies.
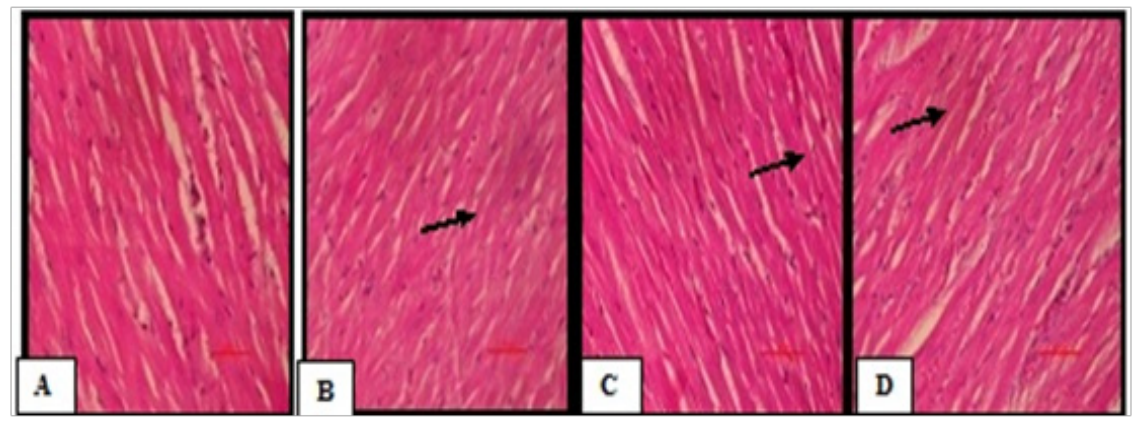

Figure 7 (A-D) Histopathology of the Heart: Representative photographs demonstrating myocardial tissue sections stained with H\&E among various experimental groups.

A: Normal control- Normal architecture of the myocardium;

B: Diabetic -Isoproterenol control-Marked edema, confluent areas of necrosis and separation of myofibers, congested blood vessels, inflammation and haemorrhage;

C:Vildagliptin - Less inflammation, edema was observed;

D: Terminalia arjuna -Occasional focal myofiber loss, less inflammation, necrosis and edema was observed.Arrows indicate separation of myofibers congested blood vessels inflammation.

Scale bar $=100 \mu \mathrm{m}$

\section{Histopathological section of Liver}

Photomicrograph of the liver of the NC group (Plate A) rats, showed normal architecture of central vein, peripheral vein and hepatocytes. In contrast, the liver cells of the D-ISP group (Plate B) showed degeneration, scattered necrotic cells, congestion in the central vein as compared to NC group. However VIL treatment (Plate C) decreased the granular degeneration as compared to D-ISP rats. In TA treated group (Plate D) mild granular degeneration, inflammatory infiltration, edema, necrosis, hepatocytes degeneration which was less compared to standard drug group was observed (Figure 9).

\section{Histopathological section of Kidney}

Photomicrograph of NC group kidney (Plate A) showed normal structure of the kidney, absence of congestion of glomerular blood vessels, tubular necrosis and inflammation. In contrast, histological assessment of the D-ISP group (Plate B) demonstrated marked congestion of glomerular blood vessels, tubular necrosis, inflammation 
and cloudy degeneration. In VIL treated group rats kidney showed less congestion of glomerular blood vessels, inflammation and focal area
(Plate $\mathrm{C}$ ). In TA group rats, renal tissue section showed less tubular necrosis and inflammation (Plate D) (Figure 10).

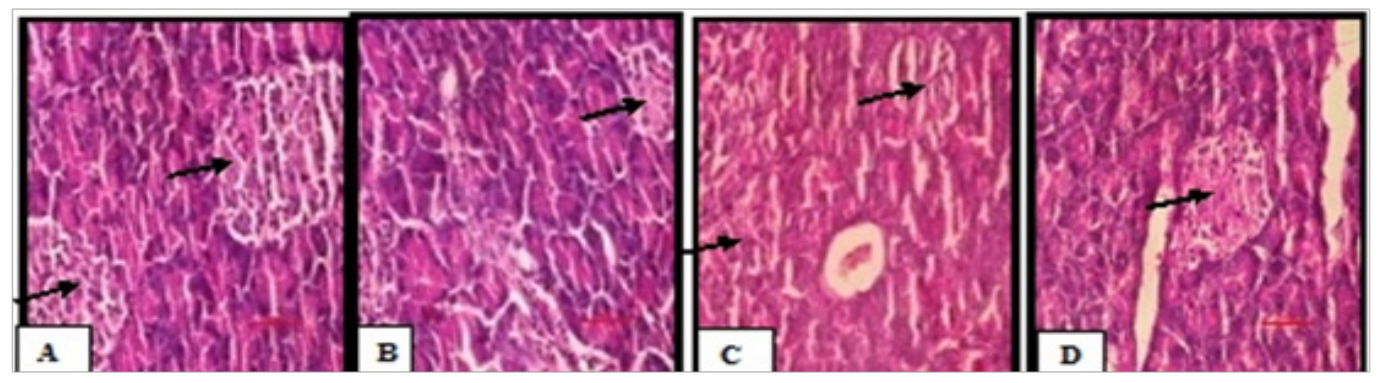

Figure 8 (A-D) Representative photographs demonstrating pancreatic tissue sections stained with H\&E among various experimental groups.

A: Normal control-Organized pattern and normal architecture of islets of langerhans and the beta cells mass;

B: Diabetic -Isoproterenol control-Damaged islets of langerhans, atrophy of beta cells and reduced beta cell mass;

C:Vildagliptin - Improved beta cell mass, less fibrosis, less inflammatory infiltration and no hemorrhage;

D: Terminalia arjuna - Improved beta cell mass, less inflammatory infiltration and no hemorrhage as compared to D-ISP group.Arrows indicate the beta cells mass.

Scale bar $=100 \mu \mathrm{m}$
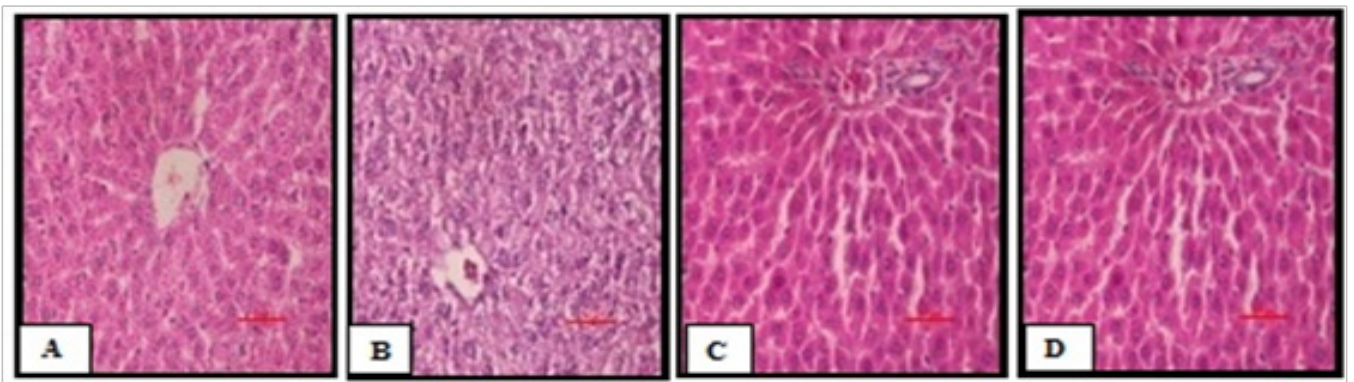

Figure 9 (A-D) Representative photographs demonstrating histopathological finding of liver tissue sections stained with H\&E among various experimental groups.

A: Normal control-Normal architecture of central vein, peripheral vein \& hepatocytes;

B: Diabetic-Isoproterenol control-scattered necrotic cells, congestion in the central vein;

C :Vildaglipti-Less granular degeneration, inflammation and necrosis;

D:Terminalia arjuna -Very Less granular degeneration, inflammation, edema, necrosis;

Scale bar $=100 \mu \mathrm{m}$.
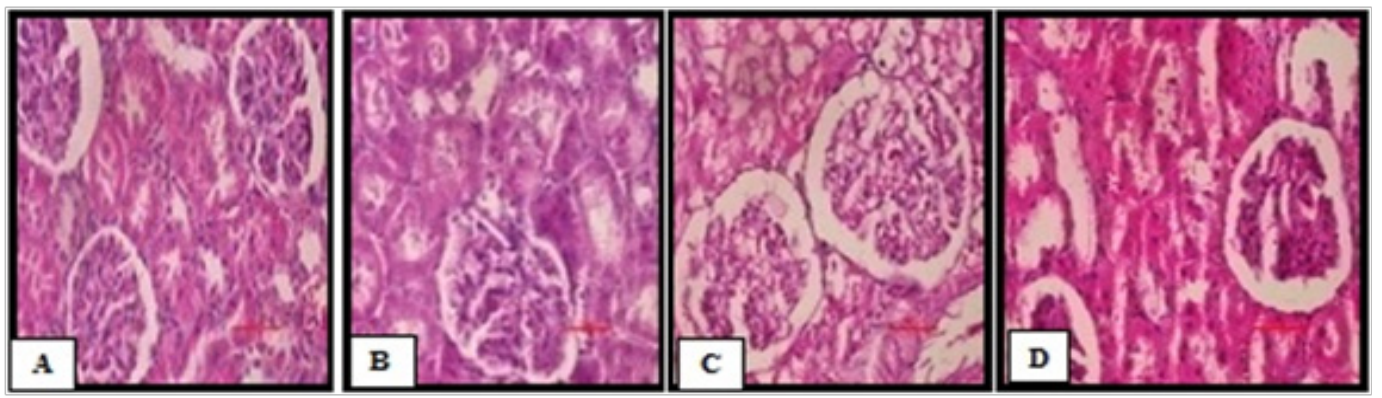

Figure 10 (A-D) Representative photographs demonstrating histopathological finding of kidney tissue sections stained with H\&E among various experimental groups.

A: Normal control-Normal structure of the kidney;

B: Diabetic-Isoproterenol control-Marked congestion of glomerular blood vessels, tubular necrosis, inflammation and cloudy degeneration;

C:Vildagliptin-Mild tubular necrosis, inflammation and edema;

D: Terminalia arjuna-less tubular necrosis and inflammation;

Scale bar $=100 \mu \mathrm{m}$.

Citation: Borde MK, Mohanty IR, Maheshwari U, et al. Natural dipeptidyl peptidase-4 inhibitor Terminalia arjuna mitigates myocardial infarction co-existing with diabetes in experimental rats. J Diabetes Metab Disord Control. 2018;5(2):48-56. DOI: I0.15406/jdmdc.20I8.05.00I37 
Table 2 Safety parameters among various experimental groups

\begin{tabular}{lllll}
\hline SNO & Variables/Groups & Pancreatic Marker Lipase(U/L) & Liver Marker SGPT(U/L) & Renal Marker Creatinine (mg/dl) \\
\hline I & NC & $30.36 \pm 1.15$ & $61.25 \pm 8.65$ & $0.32 \pm 0.07$ \\
2 & D-ISP & $42.46 \pm 4.11$ I** & $84.54 \pm 5.57 * * *$ & $0.61 \pm 0.05 * * *$ \\
4 & VIL & $38.53 \pm 3.62$ & $74.36 \pm 8.68 @ @$ & $0.48 \pm 0.07 @$ \\
5 & TA & $33.68 \pm 0.9 @ @ @ \#$ & $68.35 \pm 3.62 @ @$ & $0.39 \pm 0.05 @ @ \#$ \\
\hline
\end{tabular}

Values are expressed as mean \pm SD.

***p<0.00I NCVS D-ISP;

@@@ p<0.00 I;

@@ $\mathrm{p}<0.01$,

@ $\mathrm{p}<0.05 D-I S P V S V I L, T A ;$

\#\# $\mathrm{p}<0.01$, \#<0.05 VIL VS TA.

CPK-MB, Creatinine phosphokinase-MB; hs-CRP, High sensitive reactive protein; DPP-4, Dipeptidyl peptidase-4; NC, Normal control ( $\mathrm{n}=8$ ); D-ISP, DiabeticIsoproterenol control $(n=9) ;$ TA, Terminalia arjuna $(n=7)$

\section{Discussion}

Terminalia arjuna belongs to family Combretaceae, commonly known as Arjuna has traditionally been used in the Indian system of medicine for several medicinal purposes; cardiotonic, antidiabetic, antidysenteric, antipyretic and astringent. ${ }^{10}$ There is scientific evidence for the beneficial effects of Terminalia arjuna on diabetes mellitus and cardiovascular diseases. Previous study reported from the laboratory that Terminalia arjuna, significantly inhibits DPP-4 enzyme. ${ }^{22}$ In the present study, for the first time the cardioprotective efficacy and safety of this natural DPP-4 inhibitor Terminalia arjuna was evaluated in the setting of diabetes using an experimental model of myocardial infarction co-existing with diabetes. Terminalia arjuna therapy demonstrated significant cardioprotective effects in experimental model of myocardial infarction co-existing with diabetes. Myocardial salvaging effects of Terminalia arjuna treatment protocols may be attributed to several biochemical (Antidiabetic \{Blood glucose, $\mathrm{HbA} 1 \mathrm{c}\}$, cardiac $\{\mathrm{CPK}-\mathrm{MB}\}$, inflammatory marker $\{$ hs-CRP $\}$, DPP4 pathway $\{$ Serum DPP-4\}, safety markers \{Pancreatic function [lipase], liver function [SGPT], kidney function [Creatinine]\}. Histopathological and biochemical markers of injury confirmed the safety of Terminalia arjuna on the pancreatic, hepatic and renal functions. The present study confirmed the hypoglycaemic effects of hydroalcoholic extracts of Terminalia arjuna in diabetic rats challenged with Isoproterenol. A significant decrease in the blood glucose and HbA1c levels was observed in the VIL and TA treated groups as compared to D-ISP control group. Observed antidiabetic activity of Terminalia arjuna was supported by M. Biswas et al., ${ }^{23}$ Siddique et al., ${ }^{11}$ Ragavan B et al. ${ }^{12}$ Terminalia arjuna has been reported to increase peripheral glucose utilization and enhanced insulin action. Terminalia arjuna contains the active principles like glycosides, alkaloids, terpenoids, flavonoids etc., have antioxidant activity. Antioxidant treatment has beneficial effects on preservation of $\beta$-cell function in diabetes and also Flavonoids are known to regenerate the damaged $\beta$-cell in the alloxan induced diabetic rats. ${ }^{24,25}$ It may have exerted its beneficial effect by its antioxidant activity (Figure 2) (Figure 3). The present study for the first time reported the cardioprotective efficacy of natural DPP-4 inhibitor: Terminalia arjuna in the presence of diabetes based on various biochemical and histopathological studies. The heart to body weight ratio, is considered as an index of cardiac hypertrophy. Boluyt et al. ${ }^{26}$ demonstrated that continuous infusion of ISP in rats elicits typical cardiac gene expression and hypertrophy due to pressure overload. ${ }^{26}$ Similar to ISP administration, STZ also results in an increase in fibrous tissue and collagen, causing an increase in the heart to body weight ratio in diabetes. TA and VIL treatment resulted in a significant reduction in the heart to body weight ratio as compared to D-ISP rats. The protective effect of Terminalia arjuna on cardiac hypertrophy was supported by Santosh Kumar et al. ${ }^{27}$ In addition treatment with TA and VIL significantly restored elevated serum CPK-MB levels as compared to D-ISP group. However, VIL therapy demonstrated superior cardioprotective efficacy as compared to TA. This cardio protective finding of Terminalia arjuna was in agreement with an earlier studies reported by Shukla et al. ${ }^{28}$ and Adila Parveen et al. ${ }^{29}$ in ISP induced cardiotoxicity. (Figure 4-6), (Table 1) The protective effects of Terminalia arjuna on myocardial injury demonstrated by biochemical parameters was confirmed by histopathological assessment. Terminalia arjuna treatment resulted in structural improvement in myocardium of experimental rats. Reduced edema, inflammation and necrosis was observed as compared to D-ISP group. Such an observation was in accordance with earlier reports by Sukla et al. ${ }^{28}$ (Figure 7). In addition to cardioprotection, TA and VIL treated rats showed significant reduction in serum DPP4 levels as compared to D-ISP group; one of the mechanisms that explain their anti-hyperglycemic activity. Strikingly, the decrease in serum DPP-4 levels positively correlated with the cardiac injury marker CPK-MB (Figure 6). Thus, DPP-4 inhibition has beneficial effects on the heart. Interestingly, the DPP-4 inhibitory activity of Terminalia arjuna has been reported for the first time in the present study. DPP-4 has 3 major functions; adenosine deaminase binding, peptidase activity, and extracellular matrix binding, all of which potentially influence the activity of the immune and/or endocrine systems..$^{30}$ Although DPP-4 cleaves and inactivates several cardioactive peptides, including neuropeptide Y, BNP, SDF-1 and GLP-1. Our observations demonstrating that inhibition of DPP-4 is associated with restored elevated serum CPK-MB, suggests that DPP4 modifies cardiovascular outcomes.

The present study also evaluated the safety of standard drugs and test drugs on the vital organs: pancreas, liver and kidney. The markers of pancreatic function (pancreatic lipase), liver function 
(SGPT), kidney function (Creatinine) were assessed in addition to histopathological evaluation of the degree of injury. Increased pancreatic lipase levels as seen in D-ISP rats showed presence of pancreatic tissue damage. This was attenuated by various treatment protocols, there by restoring the architecture of the pancreas. This is the first report of the effect of test drugs Terminalia arjuna on the pancreatic function in the experimental model of myocardial infarction in setting of diabetes. Terminalia arjuna therapy did not adversely affect the hepatic and kidney function as evidenced by liver and renal function biochemical markers as well as histopathological studies. Earlier report by Chanan kumar et al. ${ }^{31}$ supported that Terminalia arjuna does not adversely affect liver and kidney function. Evidence from several studies have suggested that DPP-4 inhibitors improve cardiac function in both animal and clinical studies. ${ }^{32-34}$ In order to delineate if cardioprotective effects of Terminalia arjuna is attributed to DPP-4 inhibition, Serum DPP-4 levels were estimated in the various experimental groups. Terminalia arjuna and Vildagliptin treatment, restored the elevated DPP-4 levels observed in the D-ISP rats. Vildagliptin treatment showed superior reduction in serum DPP-4 levels as compared to Terminalia arjuna. It was also found that the cardioprotection (as indicated by cardiac marker CPK-MB levels) demonstrated by Terminalia arjuna and Vildagliptin was found to positively correlate with serum DPP-4 levels indicating that modulation the DPP-4 pathway contributes to their cardioprotective efficacy. Thus, the present study demonstrated the DPP-4 inhibition contributes to the myocardial salvaging effects of Terminalia arjuna in the setting of diabetes.

\section{Conclusion}

Terminalia arjuna demonstrated beneficial effects in experimental model of myocardial infarction co-existing with diabetes.

\section{Acknowledgement}

None

\section{Conflict of interest}

None

\section{References}

1. Go AS, Mozaffarian D, Roger VL, et al. Executive summary: heart disease and stroke statistics--2013 update: a report from the American Heart Association. Circulation. 2013;127(1):143-152.

2. Tancredi M, Rosengren A, Svensson AM, et al. Excess Mortality among Persons with Type 2 Diabetes. N Engl J Med. 2015;373(18):1720-1732.

3. Scheen AJ. Cardiovascular effects of dipeptidyl peptidase-4 inhibitors from risk factors to clinical outcomes. Postgrad Med. 2013;125(3):7-20.

4. Fisman EZ, Tenenbaum A. Antidiabetic treatment with gliptins: focus on cardiovascular effects and outcomes. Cardiovasc Diabetol. 2015;14(129):1-13

5. L Ferreira, E Teixeira De Lemos, F Pinto et al. Effects of sitagliptin treatment on dysmetabolism, inflammation, and oxidative stress in an animal model of type 2 diabetes (ZDF rat). Mediators of Inflammation. 2010:1-36.

6. Matteucci E, Giampietro O. Dipeptidyl peptidase-4 inhibition: linking chemical properties to clinical safety. Curr Med Chem. 2011;18(31):4753-4760.
7. Nicolau Ramis J, Masmiquel Comas L. The new antidiabetic Agents in firing line. safety reason or witch hunt. Endocrinol Nutr. 2012;59(1):1-

8. Monami M, Dicembrini I, Mannucci E. Dipeptidyl peptidase-4 inhibitors and pancreatitis risk: a meta-analysis of randomized clinical trials. Diabetes Obes Metab. 2014;16(1):48-56.

9. Elashoff M, Matveyenko AV, Gier B, et al. Pancreatitis and Thyroid Cancer with Glucagon like Peptide-1-based Therapies. Gasteroenterology. 2011;141(1):150-156.

10. Amalraj A, Gopi S. Medicinal properties of Terminalia arjuna (Roxb.) Wight \& Arn.: A review. Journal of Traditional and Complementary Medicine. 2016;7(1): 65-78.

11. Kehkashan Parveen, Rashid Khan, Waseem A Siddiqui. Antidiabetic effects afforded by Terminalia arjuna in high fat-fed and streptozotocininduced type 2 diabetic rats. Int J Diabetes \& Metab. 2011;19:23-33.

12. Ragavan B, Krishnakumari S. Effect of T. arjuna stem bark extract on histopathology of liver, kidney and pancreas of alloxan-induced diabetic rats. Afr J Biomed. 2006;9:189-197.

13. Barman S, Das S. Hypoglycemic effect of ethanolic extract of bark of Terminalia arjuna Linn. in normal and alloxan-induced noninsulindependent diabetes mellitus albino rats. Int $J$ Green Pharm. 2012;6(4):279-284.

14. Manjunath S, Kugali SN, Deodurg PM. Effect of clonidine on blood glucose levels in euglycemic and alloxan-induced diabetic rats and its interaction with glibenclamide. Indian J Pharmacol. 2009;41(5):218220.

15. GS George, AA Uwakwe. Hypoglycemic Properties of Some Local Herbs Extracts in Streptozotocin-Induced Diabetic Wistar Albino Rats. Journal of Dental and Medical Sciences. 2014;13(1):1-7.

16. Viswanatha Swamy AH, Patel UM, Koti BC, et al. Cardioprotective effect of Saracaindicaagainst cyclophosphamide induced cardiotoxicity in rats: A biochemical, electrocardiographic and histopathological study. Indian J Pharmacol. 2013;45(1):44-48.

17. Al-masri IM, Mohammad MK, Tahaa MO. Inhibition of dipeptidyl peptidase IV (DPP-IV) is one of the mechanisms explaining the hypoglycemic effect of berberine. J Enzyme Inhibi Medicinal Chemistry. 2009;24(5):1061-1066.

18. Marcy EM, Hayes TE, Tracy RP. Variability in measurement of C-reactive protein in healthy subjects: implication reference interval and epidemiological applications. Clin Chem.1997;43(1):52-58.

19. Imamura S, Hirayama $T$, Arai $T$, et al. An enzymatic method using 1, 2-diglyceride for pancreatic lipase test in serum. Clin Chem.1989;35:1126-1132.

20. King EJ. 2-4DNPH method of determination of serum GOT and GPT. Can Med Assoc J. 1934;31:326.

21. Jaffe M. Ueber den Neiderschlag, welchenPikrinsäureimnormalenharn Erzeught und übereineneue Reaction des Kreatinins. Z Physiol Chem.1886;10(5):391-400.

22. Manjusha $\mathrm{k}$ borde, Ipseeta ray mohanty, Rajesh kumar suman, et al. dipeptidyl peptidase-iv inhibitory activities of medicinal plants: terminalia arjuna, commiphora mukul, gymnema sylvestre, morinda citrifolia, emblica officinalis. Asian J Pharm Clin Res. 2016;9(3):180182 .

23. Moulisha Biswas, Biswakanth Kar, Sanjib Bhattacharya, et al. Antihyperglycemic activity and antioxidant role of Terminalia arjuna leaf in streptozotocin-induced diabetic rats. Pharmaceutical Biology. 2011;49(4):335-340. 
24. Kaneto H, Kajimoto Y, Miyagawa J, et al. Beneficial effects of antioxidants in diabetes:Possible protection of pancreatic b-cells against glucose toxicity.Diabetes 1999;48(12):2398-2406.

25. Hakkim FL, Girija S, Kumar RS, et al. Effect of aqueous and ethanol extracts of Cassia auriculata L. flowers on diabetes using alloxan induced diabetic rats. Int J Diabetes and Metabolism. 2007;15:100-106.

26. Boluyt MO, Long X, Eschenhagen T, et al. Isoproterenol infusion induces alterations in expression of hypertrophy-associated genes in rat heart. Am J Physiol. 1995;269(2):638-647.

27. Santosh Kumar, Pankaj Prabhakar, Subir K Maulik, et al. Terminalia arjuna limits $\beta$-adrenergic receptors mediated cardiac hypertrophy in vivo. J Clin Exp Cardiolog.2013;4:4155-4158

28. Shukla, Santosh K Sharma, Suman B Singh, et al. Terminalia arjuna wight and arnn aguments cardioprotection via antioxidant and antiapoptocic cascaidein isoproterenol induced cardiotoxicity in rats. Indian journal of experimental biology. 2015;53:810-818.

29. Adila Parveen, Rashmi Babbar, Sarita Agarwal, Anita Kotwani, Mohammad Fahim.Mechanistic Clues in the Cardioprotective Effect of Terminalia Arjuna Bark Extract in Isoproterenol-Induced Chronic Heart Failure in Rats. Cardiovasc Toxicol. 2011;11(1):48-57.
30. Aytac U, Dang NH : CD26/dipeptidyl peptidase IV: a regulator of immune function and a potential molecular target for therapy. Curr Drug Targets Immune Endocr Metabol Disord.2004;4(1):11-18.

31. Connelly KA, Zhang Y, Advani A, et al. DPP-4 inhibition attenuates cardiac dysfunction and adverse remodeling following myocardial infarction in rats with experimental diabetes. Cardiovasc Ther. 2013;31(5):259-267.

32. Chandan Kumar,Raj kumar,Samshun Nehar. Hydroglycemic effects of Terminalia arjuna roxb bark on type- 2 diabetic albino rat. The Bioscience. 2013;8(2):709-712.

33. Read PA, Khan FZ, Dutka DP. Cardioprotection against ischaemia induced by dobutamine stress using glucagon-like peptide-1 in patients with coronary artery disease. Heart. 2012;98(5):408-413.

34. Connelly KA, Zhang Y, Advani A, et al. Gilbert DPP-4 Inhibition Attenuates Cardiac Dysfunction and Adverse Remodeling Following Myocardial Infarction in Rats with Experimental Diabetes. Cardiovascular Therapeutics. 2013;31(5):259-267. 